Palavras chave:

Modelo estocástico

Covariável

Multiproduto

Histórico:

Recebido I //10/201 I

Aceito 29/04/2014

Keywords:

Probability models

Covariates

Multi-products

Correspondência: adriano.mendonca@ufes.br
Adriano Ribeiro de Mendonça', Natalino Calegario², Gilson Fernandes da Silva', Agostinho Lopes de Souza ${ }^{3}$, Paulo Fernando Trugilho², Samuel Pádua Chaves e Carvalho ${ }^{4}$, Ernani Lopes Possato ${ }^{2}$

\section{MODELAGEM DA PRODUÇÃO DE SORTIMENTOS EM POVOAMENTOS DE EUCALIPTO}

RESUMO: Objetivou-se, no presente trabalho, avaliar modelos para estimar a produção de sortimentos de plantios clonais do híbrido Eucalyptus camaldulensis x Eucalyptus urophylla com espaçamento $3 \times 3$ m, localizados em Paraopeba, Minas Gerais, Brasil. Para estimar a produção em área basal e volume dos sortimentos de povoamentos, foram utilizados dados parcelas permanentes. Para estimar o volume dos sortimentos, foram analisados o modelo logístico com adição de covariáveis e o modelo de Clutter. Para a seleção do modelo mais preciso, foram utilizadas as estatísticas erro padrão relativo [Syx $(\%)]$, viés $(V)$, média das diferenças (MD), desvio padrão das diferenças (DPD) e análise gráfica dos resíduos. De acordo com as análises, o modelo Logístico obteve o melhor resultado para estimar a produção em volume de madeira para energia e celulose. A metodologia de estimação da produção de sortimentos de plantações florestais analisada foi de fácil aplicação e precisa.

\section{MODELING OF ASSORTMENT YIELDS IN EUCALYPTUS STANDS}

ABSTRACT: The aim of this study was to evaluate models to estimate the assortment yield of clonal Eucalyptus camaldulensis $\mathrm{x}$ Eucalyptus urophylla hybrid plantations with $3 \times 3 \mathrm{~m}$ spacing in the Paraopeba city, Minas Gerais, Brazil. To estimate basal area and volume of the assortment yield data from permanent plots was used. To estimate the volume of assortments, we analyzed the logistic model with covariates and the Clutter model. The statistical standard error [Syx (\%)], bias (V), average of the differences (MD), and standard deviation of the differences (DPD), as well as the graphical analysis of the residuals were used to select the most accurate model. According to the analysis, the Logistic model obtained the best results for estimation of the yield in wood volume for energy and pulpwood. The methodology to estimate assortment of forest plantations was considered easy to use and accurate. 


\section{INTRODUÇÃO}

foco das empresas florestais tem se voltado para a obtenção de multiprodutos em seus povoamentos. Com isso, as empresas, antes voltadas à produção de matéria-prima para um determinado mercado consumidor, passam a obter produtos diferenciados durante a rotação dos povoamentos. Uma característica importante nessa visão é a obtenção de receitas intermediárias durante o ciclo da cultura.

A utilização das técnicas de modelagem é de grande aplicação na quantificação de multiprodutos nos povoamentos. Essa quantificação depende da estimatição do diâmetro e/ou altura e volume em qualquer porção do tronco. A obtenção de diâmetros relativos a diversas alturas comerciais e o volume das seções do tronco é feita a partir de equações de afilamento, perfil ou taper. A utilização de modelos para representação e explicação de uma realidade essencialmente mais complexa constitui estratégia de grande valia para 0 desenvolvimento da ciência, nos diferentes campos de conhecimento (HASENAUER, 2000).

Aliado a essa necessidade de obtenção do volume de sortimentos, também é necessário avaliar - comportamento do crescimento e da produção desses sortimentos no povoamento. Essa necessidade é decorrente da importância dessa variável no planejamento de empresas do setor florestal, uma vez que toda a estrutura de manejo, transporte e comercialização depende do volume dos diferentes tipos de sortimentos. Muitos estudos foram feitos para estimar o crescimento e da produção de volume do povoamento, podendo citar: Abreu et al. (2002), Buckman (1962), Calegario et al. (2004), Clutter (1963), Schumacher (1939), Scolforo e Machado (1996), Vaccaro et al. (2003) e Wendling et al. (1998); dentre outros. Mas nenhum dos supracitados estudaram o comportamento dos sortimentos em relação à idade do plantio.

Alguns trabalhos utilizaram a modelagem da distribuição diamétrica e posterior emprego de uma equação de afilamento ou perfil do tronco para estimar a produção de sortimentos. Nessa linha de pesquisa podem ser citados os trabalhos de Acerbi Júnior et al. (2002), Gomes et al. (1997) e Sanquetta et al. (2004). A modelagem da distribuição diamétrica baseia-se no uso de funções densidade de probabilidade (f.d.p.); relações hipsométricas; modelos para estimar a mortalidade, modelos para estimar diâmetro mínimo, diâmetro máximo, diâmetro médio e variância dos diâmetros. Em razão desse grande número de variáveis a serem estimadas, essa metodologia se torna trabalhosa. Além disso, algumas dessas variáveis não são estimadas com grande precisão, acarretando em estimativas com pouca confiabilidade.

Para facilitar a modelagem da produção de sortimentos ao longo do tempo, pode-se trabalhar com modelos que estimam a produção destes em função da idade do povoamento, incluindo os efeitos que afetam a produção do povoamento como a área basal e o índice de sítio. Esses modelos baseiam-se em informações de fácil medição e boa precisão nos processos de estimação. Nesse contexto, objetivou-se, no presente trabalho, avaliar modelos para estimar a produção do volume de sortimentos em povoamentos de Eucalyptus camaldulensis x Eucalyptus urophylla.

\section{MATERIAL E MÉTODOS}

\section{Fonte de dados}

Os dados utilizados foram obtidos numa área pertencente a $V$ \& $M$ Florestal, no município de Paraopeba, Minas Gerais, Brasil. A temperatura média da região é de $20,9^{\circ} \mathrm{C}$ e precipitação anual de 1328,7 mm (INSTITUTO BRASILEIRO DE GEOGRAFIA E ESTATÍSTICA - IBGE, 20I0). Foram utilizados dados de plantios clonais de Eucalyptus camaldulensis $\times$ Eucalyptus urophylla no espaçamento $3 \times 3 \mathrm{~m}$. Para estimar a produção em área basal e volume dos sortimentos dos povoamentos, foram utilizados dados de diâmetro a I,30 m (DAP), altura total $(\mathrm{H})$ medidos em parcelas permanentes. Após a cubagem rigorosa de 46 árvores, foi gerada a distribuição diamétrica das árvores-amostra medidas para estimar volumes individuais, apresentada na Tabela I.

TABELA I Distribuição diamétrica das árvores-amostra utilizadas no ajuste dos modelos de perfil de Eucalyptus camaldulensis x Eucalyptus urophylla.

TABLE I Diameter distribution of the sample-trees used for fitting the profile models of Eucalyptus camaldulensis $x$ Eucalyptus urophylla.

\begin{tabular}{cccccc}
\hline \multirow{2}{*}{$\begin{array}{c}\text { Centro de classe } \\
\text { de diâmetro }(\mathrm{cm})\end{array}$} & \multicolumn{6}{c}{ Centro de classe de altura $(\mathrm{m})$} & Total \\
\cline { 2 - 6 } & 12,5 & 17,5 & 22,5 & 27,5 & \\
\hline 7,5 & 3 & 12 & 1 & - & 16 \\
12,5 & 2 & 5 & 9 & - & 16 \\
17,5 & - & - & 9 & 1 & 10 \\
22,5 & - & - & 2 & 2 & 4 \\
\hline Total & 5 & 17 & 21 & 3 & 46 \\
\hline
\end{tabular}




\section{Estimação do perfil e cálculo do volume de sortimentos}

Os sortimentos (s) analisados foram: I - energia (porção da árvore menor que $7 \mathrm{~cm}$ de diâmetro) e 2 celulose (porção da árvore com diâmetro mínimo maior ou igual a $7 \mathrm{~cm})$.

Para estimar os sortimentos das árvores, foi ajustado o modelo de Schöepfer modificado representado pela equação $\mathrm{I}$, sendo $\mathrm{d}_{\mathrm{ij}}=$ diâmetro na altura $h_{i j}(m) ; D P_{j}=$ diâmetro na altura de I,30 m (m) da árvore $\mathrm{j} ; \mathrm{H}_{\mathrm{j}}=$ altura total da árvore $\mathrm{j}(\mathrm{m}) ; \mathrm{h}_{\mathrm{ij}}=$ altura comercial $(\mathrm{m})$ na posição i da árvore $\mathrm{j} ; \beta_{0}, \beta_{1}, \beta_{2}, \beta_{3}, \beta_{4}$, $\beta_{5}$ e $\beta_{6}=$ parâmetros da regressão.

O volume dos sortimentos foi obtido por meio da integral da função de afilamento (equação 2), cuja solução está apresentada na equação 3 , sendo $\mathrm{v}_{\mathrm{sj}}=$ volume do sortimento $\mathrm{s}$ da árvore $\mathrm{j}\left(\mathrm{m}^{3}\right) ; \mathrm{c}_{0}=\beta_{0}$; $c_{1}=\beta_{1} \cdot D A P_{i} ; c_{2}=\left(\beta_{2} \cdot \frac{D A P_{i}}{H_{j}}\right) ; c_{3}=\left(\beta_{3} \cdot \frac{D A P_{i}}{H_{j}^{2}}\right) ; c_{4}=$ $\left(\beta_{4} \cdot \frac{D^{2} P_{j}}{H_{j}^{3}}\right) ; c_{5}=\left(\beta_{5} \cdot \frac{D A P_{j}}{H_{j}^{4}}\right) ; c_{6}=\left(\beta_{6} \cdot \frac{D A P_{j}}{H_{j}^{5}}\right) ; h_{1}$ e $h_{2}=$ limites da integração).

A altura onde ocorre o diâmetro comercial do sortimento $\left(\mathrm{h}_{2}\right)$ foi obtida por meio do modelo Logístico com adição das covariáveis raio a l,30 m do solo e altura total nos parâmetros (equação 4), sendo rap $_{j}=$ raio a $\mathrm{I}, 30 \mathrm{~m}$ de altura da árvore $\mathrm{j}(\mathrm{m}) ; \mathrm{r}_{\mathrm{ij}}=$ raio na altura $\mathrm{h}_{\mathrm{ij}}(\mathrm{m})$.
Modelagem do crescimento e produção volumétrica

A escolha dos modelos foi baseada no estudo do comportamento do crescimento do volume por sortimento em função da idade. Na Figura I, apresentase o comportamento do volume dos sortimentos, em função da idade do povoamento.
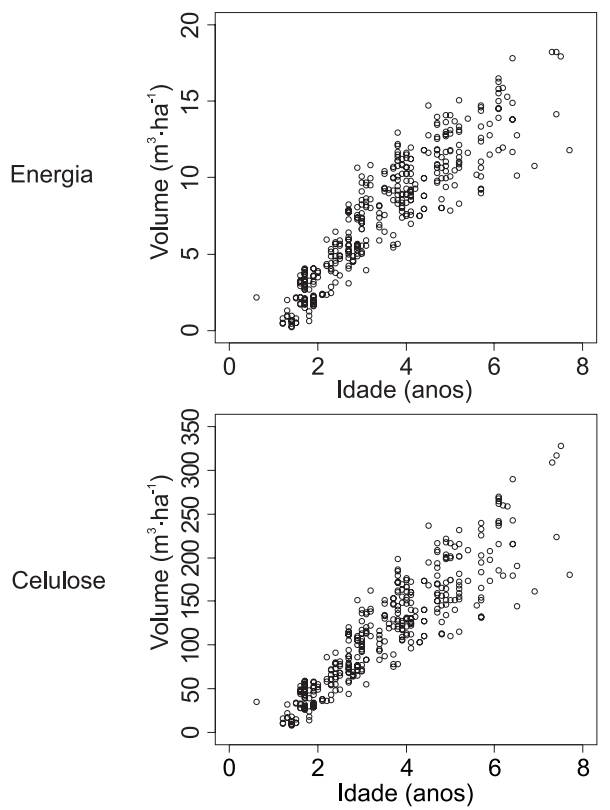

FIGURA I Comportamento do volume de madeira para energia e celulose em função da idade do povoamento.

FIGURE I Behavior of the volume of wood for energy and pulpwood according to the age of the stand.

$$
\begin{aligned}
& \mathrm{d}_{\mathrm{ij}}=\beta_{0}+\beta_{1} \cdot \mathrm{DAP}+\beta_{2} \cdot\left(\frac{\mathrm{h}_{\mathrm{ij}}}{\mathrm{H}_{\mathrm{j}}}\right) \cdot D A P_{\mathrm{j}}+\beta_{3} \cdot\left(\frac{\mathrm{h}_{\mathrm{ij}}}{\mathrm{H}_{\mathrm{j}}}\right)^{2} \cdot D A P_{j}+\beta_{4} \cdot\left(\frac{\mathrm{h}_{\mathrm{ij}}}{\mathrm{H}_{\mathrm{j}}}\right)^{3} \cdot D A P_{j}+\beta_{5} \cdot\left(\frac{\mathrm{h}_{\mathrm{ij}}}{\mathrm{H}_{\mathrm{j}}}\right)^{4} \cdot D A P_{\mathrm{j}}+\beta_{6} \cdot\left(\frac{\mathrm{h}_{\mathrm{ij}}}{\mathrm{H}_{\mathrm{j}}}\right)^{5} \cdot D A P_{j}+\varepsilon_{\mathrm{ij}} \\
& v_{s j}=\int_{h_{1}}^{h_{2}} \frac{\pi}{40.000} d_{i j}^{2} \partial h \\
& v_{s j}=\frac{\pi}{40000} \cdot\left\{\frac{c_{6}^{2} \cdot h_{i j}^{11}}{11}+\frac{c_{5} \cdot c_{6} \cdot h^{10}}{5}+\frac{\left(2 \cdot c_{6} \cdot c_{4}+c_{5}^{2}\right) \cdot h^{9}}{9}+\frac{\left(2 \cdot c_{4} \cdot c_{5}+2 \cdot c_{6} \cdot c_{3}\right) \cdot h^{8}}{8}+\frac{\left(2 \cdot c_{3} \cdot c_{5}+2 \cdot c_{6} \cdot c_{2}+c_{4}^{2}\right) \cdot h^{7}}{7}+\right. \\
& +\frac{\left[2 \cdot c_{2} \cdot c_{5}+2 \cdot c_{4} \cdot c_{3}+2 \cdot c_{6}\left(c_{0}+c_{1}\right)\right] \cdot h^{6}}{6}+\frac{\left[c_{3}^{2}+2 \cdot c_{5} \cdot\left(c_{0}+c_{1}\right)+2 \cdot c_{2} \cdot c_{4}\right] \cdot h^{5}}{5}+\frac{\left[2 \cdot c_{4}\left(c_{0}+c_{1}\right)+2 \cdot c_{2} \cdot c_{3}\right] \cdot h^{4}}{4}+ \\
& \left.+\frac{\left[c_{2}^{2}+2 \cdot c_{3}\left(c_{0}+c_{1}\right)\right] \cdot h^{3}}{3}+\left(c_{0}+c_{1}\right) \cdot c_{2} \cdot h^{2}+\left(c_{0}+c_{1}\right)^{2} \cdot h\right\}_{h_{1}}^{h_{2}} \\
& h_{i j}=\frac{\beta_{00}+\beta_{01} \cdot \operatorname{rap}_{j}+\beta_{02} \cdot H_{j}}{1+\exp \left\{\frac{\left[\left(\beta_{10}+\beta_{11} \cdot \operatorname{rap}_{j}+\beta_{12} \cdot H_{j}\right)-\frac{r_{i j}}{r a p} \cdot H_{j}\right]}{\left(\beta_{20}+\beta_{21} \cdot \operatorname{rap}_{j}\right)}\right\}}+\varepsilon_{i j}
\end{aligned}
$$


Os modelos avaliados para cada sortimento foram:

a) Logístico

O modelo logístico está apresentado na equação 5 , sendo $\mathrm{V}_{\text {sk }}=$ volume do sortimento $\mathrm{s}$ do povoamento $\mathrm{k}\left(\mathrm{m}^{3} \cdot \mathrm{ha}^{-1}\right) ; \mathrm{l}_{\mathrm{k}}=$ idade do povoamento $\mathrm{k}$ (anos); $\exp =$ base do logaritmo neperiano; $\beta_{0}, \beta_{1}$, e $\beta_{2}=$ parâmetros do modelo; e $\varepsilon_{\mathrm{sk}}=$ erro estocástico.

$\mathrm{V}_{\mathrm{sk}}=\frac{\beta_{0}}{1+\exp \left[\frac{\left(\beta_{1}-I_{\mathrm{k}}\right)}{\beta_{2}}\right]}+\varepsilon_{\mathrm{sk}}$

Baseando-se no fato de que a variação do volume dos sortimentos de povoamentos não é apenas explicada pela idade, os parâmetros do modelo logístico (5) foram decompostos, associando aos mesmos, variáveis relativas ao povoamento (índice de sítio e área basal do povoamento), por meio do modelo apresentado na equação 6 , sendo $S=$ índice de sítio do povoamento $\mathrm{k}(\mathrm{m})$; e $\mathrm{G}_{\mathrm{k}}=$ área basal do povoamento $\mathrm{k}\left(\mathrm{m}^{2} \cdot \mathrm{ha}^{-1}\right)$.

$v_{s k}=\frac{\beta_{00}+\beta_{01} \cdot S_{k}+\beta_{02} \cdot G_{k}}{1+\exp \left[\frac{\left(\beta_{10}+\beta_{11} \cdot S_{k}+\beta_{12} \cdot G_{k}\right)-I_{i}}{\left(\beta_{20}+\beta_{21} \cdot S_{k}+\beta_{22} \cdot G_{k}\right)}\right]}+\varepsilon_{s k}$

b) Clutter (1963)

O modelo de Clutter está apresentado na equação 7, sendo ln= logaritmo neperiano e os demais semelhantes à equação 5 .

$\ln \left(V_{s k}\right)=\beta_{0}+\beta_{1} \cdot\left(\frac{1}{I_{k}}\right)+\beta_{2} \cdot S_{k}+\beta_{3} \cdot \ln \left(G_{k}\right)+\varepsilon_{s k}$

Para ajuste dos modelos, foi utilizado o software estatístico $R$ versão 2.10.I (R DEVELOPMENT CORE TEAM, 20I I).

\section{Avaliação dos modelos}

Os modelos utilizados tiveram sua precisão testada por meio do erro padrão relativo, descrito nas equações 8 e 9, sendo $S_{y x}=$ erro padrão da residual; $\mathrm{S}_{\mathrm{yx}}(\%)=$ erro padrão relativo (\%); $\hat{Y}=$ valor estimado pelo modelo; $\mathrm{Y}=$ valor observado; $\bar{Y}=$ média de $\mathrm{Y} ; \mathrm{n}$ = número de observações; $\mathrm{p}=$ número de parâmetros.

$S_{y x}=\sqrt{\frac{\sum_{i=1}^{n}(Y-\hat{Y})^{2}}{n-p}}$

$S_{y x}(\%)=\frac{S_{y x}}{\bar{Y}} \cdot 100$
Adicionalmente, foram calculadas as estatísticas viés (V), média das diferenças (MD) e desvio padrão das diferenças (DPD) (MENDONÇA et al., 2007; SOUZA et al., 2008) e as análises dos gráficos do erro (\%) em função do valor estimado pelo modelo. Para cada valor observado foi obtido o valor do erro (\%) por meio da equação 10.

$\operatorname{Erro}(\%)=100 \cdot \frac{Y-\hat{Y}}{Y}$

Em seguida, foram elaborados os gráficos do erro (\%) em função do valor estimado pelo modelo.

Para avaliar o modelo de Schöepfer modificado, foi utilizado o coeficiente de determinação ajustado $\left(\overline{R^{2}}\right)$ além do Syx (\%).

\section{RESULTADOS E DISCUSSÃO}

\section{Avaliação dos modelos de perfil}

$\mathrm{Na}$ Tabela 2, apresentam-se as estatísticas do ajuste do modelo de Schöepfer modificado.

TABELA 2 Estimativa dos parâmetros do modelo de Schöepfer modificado, respectivos erros padrão, valor calculado do teste t e nível de significância.

TABLE 2 Estimative of parameters for the modified Schöepfer model, their standard errors, calculated value of t-test and $p$-value.

\begin{tabular}{ccccc}
\hline Parâmetro & Estimativa & Erro padrão & $\mathrm{t}$ & $\mathrm{p}>|\mathrm{t}|$ \\
\hline$\beta_{0}$ & 0,34237 & 0,0910 & 3,76 & $<0,001$ \\
$\beta_{1}$ & 1,15940 & 0,0083 & 138,38 & $<0,001$ \\
$\beta_{2}$ & $-3,36870$ & 0,1674 & $-20,11$ & $<0,001$ \\
$\beta_{3}$ & 12,74601 & 1,1691 & 10,90 & $<0,001$ \\
$\beta_{4}$ & $-27,12805$ & 3,1116 & $-8,72$ & $<0,001$ \\
$\beta_{5}$ & 26,29384 & 3,4953 & 7,52 & $<0,001$ \\
$\beta_{6}$ & $-9,73045$ & 1,3946 & $-6,98$ & $<0,001$ \\
\hline
\end{tabular}

Nota-se que todos os parâmetros foram significativos $(p<0,00 \mathrm{I})$, sendo que o modelo analisado consegue explicar a variação do diâmetro $\left(\mathrm{d}_{\mathrm{ij}}\right)$ ao longo do fuste. Isso é percebido pelo alto valor de $\mathrm{R}^{2}$ que foi de 0,9887 e o valor de Syx encontrado que foi de 6,55\%. Esse valor obtido para o Syx (\%) está próximo do valor encontrado por Mendonça et al. (2007), trabalhando com o modelo de Schöepfer original em Eucalyptus sp., que foi de $7,24 \%$.

$\mathrm{Na}$ Tabela 3, apresenta-se a estimativa dos parâmetros e as estatísticas do ajuste do modelo Logístico com adição de covariáveis. Percebe-se que os parâmetros do modelo Logístico foram significativos $(p<0,00$ I). 
TABELA 3 Estimativa dos parâmetros do modelo de Logístico com covariáveis, respectivos erros padrão, valor calculado do teste $t$ e nível de significância.

TABLE 3 Estimative of parameters for the Logistic model with covariates, their standard errors, calculated value of t-test and $p$-value.

\begin{tabular}{ccccc}
\hline Parâmetro & Estimativa & Erro padrão & $t_{c}$ & $\mathrm{p}>|\mathrm{t}|$ \\
\hline$\beta_{00}$ & $4,0485 \mathrm{I}$ & 0,7749 & 5,22 & $<0,00 \mid$ \\
$\beta_{01}$ & $35,4658 \mid$ & 10,4399 & 3,40 & $<0,00 \mid$ \\
$\beta_{02}$ & $0,687 \mid 3$ & $0,056 \mid$ & $\mid 2,26$ & $<0,00 \mid$ \\
$\beta_{10}$ & $-2,58670$ & 0,4465 & $-5,79$ & $<0,00 \mid$ \\
$\beta_{11}$ & $-27,94969$ & 5,9858 & $-4,67$ & $<0,00 \mid$ \\
$\beta_{12}$ & 0,83086 & 0,0325 & 25,54 & $<0,00 \mid$ \\
$\beta_{20}$ & $-2,48225$ & $0,|80|$ & $-\mid 3,78$ & $<0,00 \mid$ \\
$\beta_{21}$ & $-\mid 5,97885$ & $2,535 \mid$ & $-6,30$ & $<0,00 \mid$ \\
\hline
\end{tabular}

O modelo Logístico apresentou Syx igual a $0,77 \mathrm{~m}$ ou $9,23 \%$. O valor obtido para estatística Syx quando comparado ao trabalho de Pires e Calegario (2007) que obtiveram valores variando de I,06 m a I,28 $\mathrm{m}$ em diferentes classes diamétricas, podem ser considerados satisfatórios na estimação do afilamento das árvores de do híbrido Eucalyptus camaldulensis $\mathbf{x}$ Eucalyptus urophylla.

\section{Avaliação dos modelos para estimar o volume de sortimentos}

$\mathrm{Na}$ Tabela 4, são apresentadas as estatísticas de ajuste dos modelos Logístico com covariáveis e Clutter para estimar o volume de madeira para energia.

$\mathrm{Na}$ Tabela 4, observa-se que todos os parâmetros do modelo de Clutter foram significativos e, o parâmetro $\beta_{20}$, do modelo Logístico, não foi significativo. Mas, analisando o Syx (\%), V, MD e DPD, nota-se que o modelo Logístico teve um erro menor e menor tendência, sendo o mais preciso.

$\mathrm{Na}$ Figura 2, apresenta-se a distribuição dos resíduos dos modelos avaliados para estimar o volume de madeira para energia.

Pela Figura 2, visualiza-se que a variação dos erros, em sua maioria, se distribui entre $\pm 20 \%$. Percebe-se, também, que o modelo Logístico conseguiu representar melhor a variação de volume de madeira para energia. O modelo de Clutter apresentou uma forte tendência de superestimação em áreas com produção acima de, aproximadamente, $12,5 \mathrm{~m}^{3} \cdot \mathrm{ha}^{-1}$. Os resultados encontrados na análise gráfica de resíduos estão de acordo com os encontrados na Tabela 4.
TABELA 4 Estimativa dos parâmetros dos modelos para estimar o volume de madeiras para energia, respectivos erros padrão, valor calculado do teste $t$ e nível de significância

TABLE 4 Estimative of model parameters to estimate the volume of wood for energy, their standard errors, calculated value of $\mathrm{t}$-test and $\mathrm{p}$-value

Logístico

\begin{tabular}{ccccc}
\multicolumn{5}{c}{$(\mathrm{Syx}=2,83 \%, \mathrm{~V}=0,00030, \mathrm{MD}=0,15933, \mathrm{DPD}=0,21688)$} \\
\hline Parâmetro & Estimativa & Erro padrão & $\mathrm{t}$ & $\mathrm{p}>|\mathrm{t}|$ \\
\hline$\beta_{00}$ & $-10,49423$ & 0,5397 & $-19,44$ & $<0,001$ \\
$\beta_{01}$ & 0,36317 & 0,0246 & 14,78 & $<0,001$ \\
$\beta_{02}$ & 0,73403 & 0,0099 & 74,19 & $<0,001$ \\
$\beta_{10}$ & $-2,34267$ & 0,3651 & $-6,42$ & $<0,001$ \\
$\beta_{11}$ & 0,18612 & 0,0183 & 10,17 & $<0,001$ \\
$\beta_{12}$ & $-0,13939$ & 0,0211 & $-6,62$ & $<0,001$ \\
$\beta_{20}$ & $-0,19439$ & 0,3454 & $-0,56$ & 0,574 \\
$\beta_{21}$ & 0,04942 & 0,0118 & 4,17 & $<0,001$ \\
$\beta_{22}$ & 0,06631 & 0,0117 & 5,66 & $<0,001$ \\
\hline \multicolumn{5}{c}{} \\
\hline
\end{tabular}

Clutter

$($ Syx $=8,83 \%, V=-0,07257, M D=0,42924$, DPD $=0,67440)$

\begin{tabular}{ccccc}
\hline Parâmetro & Estimativa & Erro padrão & $\mathrm{t}$ & $\mathrm{p}>|\mathrm{t}|$ \\
\hline$\beta_{0}$ & $-2,31083$ & 0,0673 & $-34,35$ & $<0,001$ \\
$\beta_{1}$ & $-0,51235$ & 0,0550 & $-9,32$ & $<0,001$ \\
$\beta_{2}$ & 0,01452 & 0,0015 & 9,38 & $<0,001$ \\
$\beta_{3}$ & 1,52434 & 0,0202 & 75,59 & $<0,001$ \\
\hline
\end{tabular}
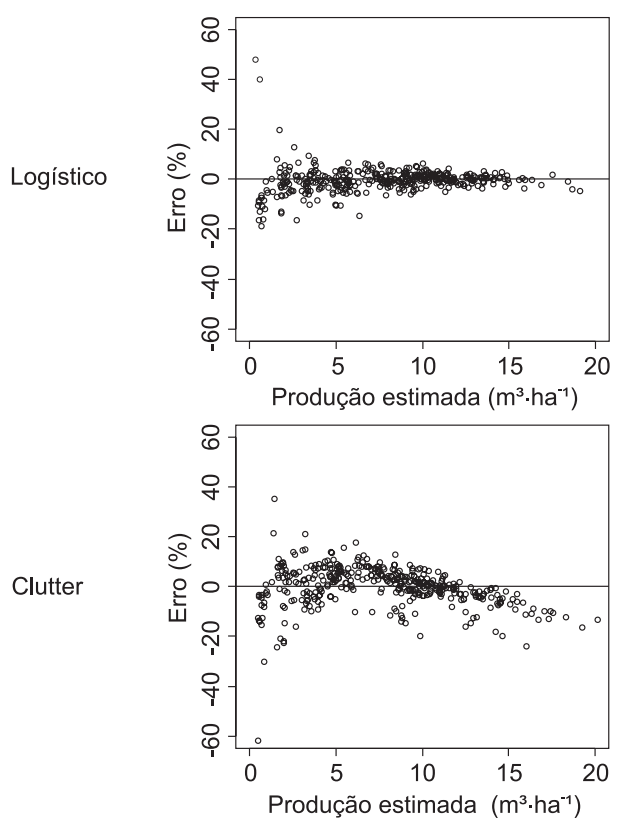

FIGURA 2 Distribuição dos resíduos, em percentagem, em função do volume estimado de madeira para energia para o modelo Logístico e modelo de Clutter.

FIGURE 2 Distribution of the residuals, in percentage, in function of estimated volume of wood for energy for the Logistic and Clutter models. 
$\mathrm{Na}$ Tabela 5, são apresentadas as estatísticas de ajuste dos modelos Logístico com covariáveis e Clutter para estimar o volume de madeira para celulose. Analisando os resultados, percebe-se que os parâmetros estimados dos modelos analisados foram significativos a $5 \%$ de probabilidade, exceto $\beta_{22}$ do modelo logístico (significativo a $10 \%$ de probabilidade). Além disso, verifica-se que o modelo Logístico obteve uma grande vantagem sobre o modelo de Clutter em termos de Syx (\%), V, MD e DPD.

TABELA 5 Estimativa dos parâmetros dos modelos para estimar o volume de madeira para celulose, respectivos erros padrão, valor calculado do teste $t$ e nível de significância

TABLE 5 Estimative of model parameters to estimate the volume of pulpwood, their standard errors, calculated value of $t$-test and $p$-value

Logístico

(Syx = 3,43\%; V = -0,0199I; MD = 2,92449; DPD = 3,88923)

\begin{tabular}{ccccc}
\hline Parâmetro & Estimativa & Erro padrão & $\mathrm{t}$ & $\mathrm{p}>|\mathrm{t}|$ \\
\hline$\beta_{00}$ & $-161,51971$ & 9,8214 & $-17,06$ & $<0,001$ \\
$\beta_{01}$ & 5,98475 & 0,4546 & $\mid 3,17$ & $<0,001$ \\
$\beta_{02}$ & 12,2954 & 0,2835 & 43,55 & $<0,001$ \\
$\beta_{10}$ & $-2,71487$ & 0,5846 & $-4,64$ & $<0,001$ \\
$\beta_{11}$ & 0,23393 & 0,0151 & 15,48 & $<0,001$ \\
$\beta_{12}$ & $-0,14403$ & 0,0254 & $-5,69$ & $<0,001$ \\
$\beta_{20}$ & 6,64710 & 0,5799 & 11,46 & $<0,001$ \\
$\beta_{21}$ & $-0,10576$ & 0,0179 & $-5,91$ & $<0,001$ \\
$\beta_{22}$ & $-0,02579$ & 0,0145 & $-1,78$ & 0,076 \\
\hline \multicolumn{5}{c}{ Clutter } \\
Syx $=6,40 \% ; \mathrm{V}=0,84207 ;$ MD $=4,89627 ; \mathrm{DPD}=7,22005)$ \\
\hline Parâmetro & Estimativa & Erro Padrão & $\mathrm{t}$ & $\mathrm{p}>|\mathrm{t}|$ \\
\hline$\beta_{0}$ & 0,75891 & 0,0474 & 16,02 & $<0,001$ \\
$\beta_{1}$ & $-0,57101$ & 0,0388 & $-14,73$ & $<0,001$ \\
$\beta_{2}$ & 0,01344 & 0,0011 & 12,33 & $<0,001$ \\
$\beta_{3}$ & 1,40058 & 0,0142 & 98,46 & $<0,001$ \\
\hline \multicolumn{5}{c}{}
\end{tabular}

Na Figura 3, está apresentada a distribuição dos resíduos dos modelos avaliados para estimar do volume de madeira para celulose.

Analisando os resultados da Figura 3, verifica-se que os modelos apresentaram erro (\%), na sua maioria, entre $\pm 20 \%$. Observa-se que o modelo logístico, para valores abaixo de $50 \mathrm{~m}^{3} \cdot \mathrm{ha}^{-1}$ apresenta erros de maiores magnitudes. A partir de $50 \mathrm{~m}^{3} \cdot \mathrm{ha}^{-1}$, o modelo Logístico apresentou boa distribuição residual. $\bigcirc$ modelo de Clutter apresenta tendência de subestimação em povoamentos acima de $250 \mathrm{~m}^{3} \cdot \mathrm{ha}^{-1}$. O modelo Logístico representou melhor a variação do verdadeiro volume de madeira
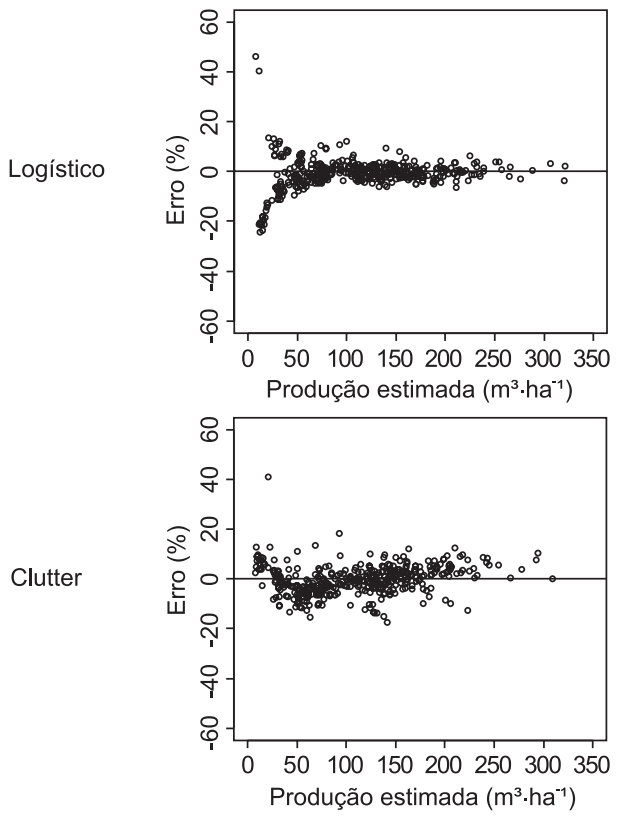

FIGURA 3 Distribuição dos resíduos, em percentagem, em função do volume estimado de madeira para celulose para o modelo Logístico e modelo de Clutter.

FIGURE 3 Distribution of the residuals, in percentage, in function of estimated volume of pulpwood for the Logistic and Clutter models.

para celulose, quando comparado à distribuição deste (Figura I). Os resultados encontrados na análise gráfica de resíduos estão de acordo com os encontrados na Tabela 5.

O melhor ajuste do modelo Logístico se deve ao seu comportamento, uma vez que este se assemelha à distribuição do volume de madeira de energia e celulose em função do tempo (Figura I). Esse modelo segue o comportamento de uma curva sigmoidal e, assim, como outros modelos não lineares, permite a obtenção dos parâmetros iniciais do modelo pela interpretação dos seus parâmetros. $O$ parâmetro $\beta_{0}$ representa a assíntota horizontal superior (AHS), isto é, o valor máximo da resposta $\left(V_{i}\right)$ no tempo $\left(I_{i}\right)$. O parâmetro $\beta_{1}$ representa o ponto de inflexão da curva, ou seja, a idade $\left(I_{i}\right)$ onde a produção $\left(V_{\mathrm{i}}\right)$ atingir metade de $\beta_{0}$. $\mathrm{E}$, o parâmetro $\beta_{2}$ (escala) representa a diferença entre as idades onde a produção atingir aproximadamente $73 \%$ de $\beta_{0}$ e a idade no ponto de inflexão. Esse modelo se apresenta como um dos de maior facilidade de interpretação. Essa facilidade é de grande importância, pois uma das maiores limitações do uso de modelos não lineares é a escolha correta dos parâmetros iniciais para o processo de iteração. Quando os valores iniciais são muito diferentes das estimativas para uma determinada base de dados, o algoritmo não converge ou geram estimativas não confiáveis (CALEGARIO et al., 2005). 
Além disso, a decomposição dos parâmetros do modelo, por meio da adição de covariáveis, permite que os valores de $\beta_{0}$, $\beta_{1}$ e $\beta_{2}$ mudem com valores diferentes da capacidade produtiva do local e da densidade expressa pela área basal do povoamento. Esse comportamento é o que ocorre no crescimento de povoamentos florestais, com isso, torna o modelo uma ferramenta adequada para estimar a produção florestal, pois o torna consistente biologicamente e aumenta a precisão de suas predições.

Apesar de ter mais parâmetros, a diferença entre os valores de Syx (\%), V, MD e DPD entre os dois modelos e dos gráficos dos resíduos, tanto para volume para energia ou celulose, corrobora o uso do modelo Logístico com covariáveis. Além disso, as estatíticas Syx (\%) e DPD penalizam os modelos com mais parâmetros, principalmente aqueles parâmetros que não melhoram as estimativas, selecionando modelos mais parcimoniosos.

\section{CONCLUSÕES}

A tendência de crescimento do volume de madeira para energia e celulose, em função do tempo, é não linear. A metodologia de estimação da produção de sortimentos de plantações florestais analisada foi precisa.

O modelo Logístico com adição de covariáveis é adequado para estimar a produção em volume de madeira para energia e celulose.

\section{AGRADECIMENTOS}

À Coordenação de Aperfeiçoamento de Pessoal de Nível Superior (CAPES) pela concessão da bolsa de estudos.

\section{REFERÊNCIAS}

ABREU, E. C. R.; SCOLFORO, J. R. S.; OLIVEIRA, A. D. de; MELLO, J. M. de; KANEGAE JÚNIOR, H. Modelagem para prognose precoce do volume por classe diamétrica para Eucalyptus grandis. Scientia Forestalis, Piracicaba, v. 6I, p. 86-I02, 2002.

ACERBI JÚNIOR, F. W.; SCOLFORO, J. R. S.; OLIVEIRA, A. D. de; MAESTRI, R. Modelo para prognose do crescimento e da produção e análise econômica de regimes de manejo para Pinus taeda L. Revista Árvore, Viçosa, v. 26, n. 6, p. 699-7I3, nov./dez. 2002.

BUCKMAN, R. E. Growth and yield of red pine in Minnesota. Washington: USDA, 1962.50 p. (Technical Bulletin, 1272).

CALEGARIO, N.; CALEGARIO, C. L. L.; MAESTRI, R.; DANIELS, R.; NEIVA, R. Melhoria da qualidade do ajuste de modelos biométricos florestais pelo emprego da teoria dos modelos não lineares generalizados. Scientia Forestalis, Piracicaba, v. 69, p. 38-50, 2005.
CALEGARIO, N.; DANIELS, R.; MAESTRI, R.; NEIVA, R. Desenvolvimento de um modelo linear de efeito misto na estimativa do crescimento e produção de povoamentos clonais de Eucalyptus. Cerne, Lavras, v. 10, n. I, p. 67-86, 2004.

CLUTTER, J. L. Compatible growth and yield models for loblolly pine. Forest Science, Bethesda, v. 9, n. 3, p. 35537I, 1963.

GOMES, F. dos S.; MAESTRI, R.; SANQUETTA, C. R. Avaliação da produção em volume total e sortimento em povoamentos de Pinus taeda $L$. submetidos a diferentes condições de espaçamento inicial e sítio. Ciência Florestal, Santa Maria, v. 7, n. I, p. I0I-I26, 1997.

HASENAUER, H. Princípios para a modelagem de ecossistemas florestais. Ciência \& Ambiente, Santa Maria, v. 20, p. 5369, 2000.

INSTITUTO BRASILEIRO DE GEOGRAFIA E ESTATÍ́STICA. Cidades. Disponível em: <http://www.ibge.gov.br>. Acesso em: 15 fev. 2010.

MENDONÇA, A. R. de; SILVA, G. F. da; OLIVEIRA, J. T. da S.; NOGUEIRA, G. S. Avaliação de funções de afilamento visando a otimização de fustes de Eucalyptus sp. para multiprodutos. Cerne, Lavras, v. I3, n. I, p. 7I-82, 2007.

PIRES, L. M.; CALEGARIO, N. Ajuste de modelos estocásticos lineares e não lineares para a descrição do perfil longitudinal de árvores. Revista Árvore, Viçosa, v. 3 I, n. 5, p. 845-852, set./out. 2007.

R DEVELOPMENT CORE TEAM. R: a language and environment for statistical computing. Vienna: R Foundation for Statistical Computing, 20II. Disponível em: <http:// www.R-project.org/>. Acesso em: 10 fev. 2013.

SANQUETTA, C. R.; REZENDE, A. V.; GAIAD, D.; SCHAAF, L. B.; ZAMPIER, A. C.; ARCE, J. E. Produção de madeira para celulose em povoamentos de Pinus taeda submetidos a diferentes densidades de plantio e regimes de desbaste: abordagem experimental. Cerne, Lavras, v. 10, n. 2, p. I54166, 2004.

SCHUMACHER, F. X. A new growth curve and its application to timber yield studies. Journal of Forestry, Bethesda, v. 37, p. 819-820, 1939.

SCOLFORO, J. R. S.; MACHADO, S. do A. Um sistema de crescimento e produção com simulador de desbaste. Scientia Forestalis, Piracicaba, v. 50, p. 51-64, 1996.

SOUZA, C. A. M. de; SILVA, G. F. da; XAVIER, A. C.; MENDONÇA, A. R. de; ALMEIDA, A. Q. de. Avaliação de modelos de afilamento não segmentados na estimação da altura e volume comercial de Eucalyptus sp. Ciência Florestal, Santa Maria, v. I8, n. 3, p. 393-405, 2008. 
VACCARO, S.; FINGER, C. A. G.;SCHNEIDER, P. R.; LONGHI, S. J. Incremento em área basal de árvores de uma floresta estacional decidual, em três fases sucessionais no município de Santa Tereza, RS. Ciência Florestal, Santa Maria, v. I3, n. 2, p. |3|-|42, 2003.
WENDLING, J. L. G.; SANQUETTA, C. R.; AHRENS, S. Modelos matemáticos para crescimento e produção de peso de creme comestível de palmito, em árvores individuais de Euterpe edulis Mart. Floresta, Curitiba, v. 28, n. 1/2, p. 65-82, 1998. 